\title{
Thmoquinone from Nigella sativa was more potent than cisplatin in eliminating of SiHa cells via apoptosis with down-regulation of Bcl-2 protein.
}

\begin{abstract}
Thymoquinone (TQ), the active constituent of Nigella sativa or black cumin exhibited cytotoxic effects in several cancer cell lines. In this study, the cytotoxicity of TQ in human cervical squamous carcinoma cells ( $\mathrm{SiHa}$ ) was investigated. TQ was cytotoxic towards $\mathrm{SiHa}$ cells with IC50 values of $10.67 \pm 0.12$ and $9.33 \pm 0.19 \mu \mathrm{g} / \mathrm{mL}$ as determined by MTT assay and trypan blue dye exclusion test, respectively, after $72 \mathrm{~h}$ of incubation. TQ was more cytotoxic towards $\mathrm{SiHa}$ cells compared to cisplatin. Interestingly, TQ was less cytotoxic towards the normal cells (3T3L1 and Vero). Cell cycle analysis performed by flowcytometer showed a significant increase in the accumulation of TQ-treated cells at sub-G1 phase, indicating induction of apoptosis by the compound. Apoptosis induction by TQ was further confirmed by Annexin V/PI and AO/PI staining. Significant elevation of p53 and down-regulation of the anti-apoptotic Bcl-2 protein was found in the treated cells, without any changes in the expression of the pro-apoptotic Bax protein. In conclusion, thymoquinone from $\mathrm{N}$. sativa was more potent than cisplatin in elimination of SiHa cells via apoptosis with down-regulation of Bcl-2 protein.
\end{abstract}

Keyword: Thymoquinone; Nigella sativa; Cervical cancer; Apoptosis; p53; Bcl-2. 